\title{
Modelagem Matemática e o consumo de energia elétrica residencial
}

\author{
Ferreira, M. V. V. ${ }^{*} ;$ Meneguelli, L ${ }^{1}$; Ferreira, A.T ${ }^{2}$; Lorenzoni , L.R ${ }^{1}$; Souza, M.A.V.F ${ }^{1}$; Rezende,L.T.R ${ }^{1}$ \\ 1 Programa de Pós-Graduação em Educação Ciências e Matemática, Instituto Federal do Espírito Santo, Vitória, ES, Brasil. \\ 2 Programa de Formação em Designer Instrucional,Instituto Federal do Espírito Santo, Vitória,ES,Brasil. \\ * e-mail: marcusvvft@gmail.com ${ }^{1}$
}

\begin{abstract}
Resumo
A Modelagem Matemática é uma metodologia de ensino que contribui para a construção de conhecimentos e produção de saber matemáticos. Modelar um problema do mundo real não é apenas executar passos sincronizados para se chegar a um resultado esperado, ou apenas memorização de conceitos, para, além disso, modelar matematicamente um problema é compreender e construir uma estratégia para solucioná-lo, executar esta estratégia e validar a solução com os argumentos utilizados. O objetivo do presente artigo é apresentar uma proposta de ensino de cálculo de consumo de energia elétrica residencial, que pode ser aplicado em turmas do ensino médio, abordando os conceitos de função afim e eletricidade.
\end{abstract}

Palavras-chave: Modelagem Matemática. Ensino de Matemática. Eletricidade.

\begin{abstract}
The mathematical modeling is a teaching methodology that contributes to the construction of knowledge and production know mathematicians. Model a real-world problem is not just perform synchronized steps to reach a desired outcome, or just memorizing concepts to further mathematically model a problem is to understand and build a strategy to fix it, execute this strategy and validate the solution with the arguments used. The purpose of this paper is to present a proposal for household electricity consumption calculation education, which can be applied in high school classes, addressing the role of concepts in order and electricity.
\end{abstract}

Keywords: Mathematical Modeling. Mathematics Teaching. Electricity.

\section{Introdução}

A Modelagem Matemática tem sido apontada por pesquisadores da Educação Matemática como uma abordagem pedagógica viável para o ensino e aprendizagem de conceitos matemáticos. Segundo Barbosa (2001), uma atividade de Modelagem está associada à problematização e investigação.

Nessa conjuntura, objetiva-se com a modelagem matemática aplicada ao tema de consumo de energia elétrica, construir um ambiente em que o aluno possa vivenciar a aplicabilidade dos conteúdos matemáticos tal como função afim, ao mesmo tempo em que desenvolve a capacidade de pesquisar, de realizar trabalhos em grupo, de discutir, refletir, criticar e comunicar suas opiniões.

\section{Modelagem matemática}

Nos debates sobre o ensino de Matemática, muito se discute sobre a importância e a necessidade de se desenvolver uma educação de dimensão crítica, significativa e voltada para os interesses sociais dos alunos, valorizando uma postura investigativa, reflexiva e crítica dos mesmos. Para que isso ocorra, Skovsmose (2011) sugere o diálogo para a construção do conhecimento, e que o processo de ensino e de aprendizagem seja direcionado a problemas relevantes na perspectiva dos alunos, relacionados ao seu contexto social. Além disso, Burak e Aragão (2012) observam que a intenção do ensino de matemática deve prever a formação de um cidadão crítico e autônomo, "capaz de trabalhar em grupos, de tomar decisões diante das situações do cotidiano, da sua vida 
familiar, da sua vida profissional, um sujeito capaz de promover transformações em sua comunidade".

Estamos vivendo em uma sociedade que passa por grandes transformações, tanto sociais, quanto econômicas. Nessas situações, é importante que os alunos investiguem e busquem meios para solucionar problemas de forma contextualizada e com posicionamento crítico.

A Modelagem Matemática, segundo Barbosa (2006), contribui para essa autonomia e independência do aluno, modificando sua postura diante da Matemática e desenvolvendo um pensamento crítico e reflexivo. $O$ que permite que o mesmo experimente um processo de investigação de problemas para transformar o meio em que vive, através da proposição e análise de modelos matemáticos.

A caracterização da atividade de Modelagem se dá a partir de uma situação ou problema real. É preciso entender o problema, simplificar e estruturar a situação proposta, identificar e matematizar as variáveis, interpretar, validar e apresentar os resultados alcançados, conforme o ciclo da modelagem (Figura 1) apresentado e adaptado por Ferri (2006).

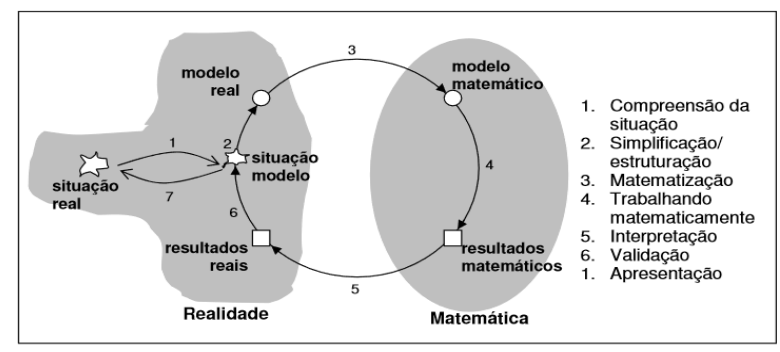

Figura 1: Ciclo da Modelagem Matemática (FERRI, 2006,p.87)

O processo de modelagem para Bassanezi (2002) consiste na arte de transformar situações da realidade em problemas matemáticos e de resolvê-los interpretando suas soluções na linguagem do mundo real. Além disso, esse processo é um caminho que torna a Matemática, em qualquer nível, mais atraente, agradável e motivadora.

\section{Consumo de Energia Elétrica}

No Brasil, a energia elétrica é gerada em sua maioria através de hidroelétricas que utilizam as águas de rios e dependem das chuvas. Para preservar nossos recursos naturais e ainda economizar na conta de energia, é necessário que haja o consumo consciente de energia elétrica. Visando promover o uso eficiente dessa energia, foi criado o Procel (Programa Nacional de Conservação de Energia Elétrica), coordenado pelo Ministério de Minas e Energia e executado pela Eletrobrás, o programa busca reduzir o desperdício de energia elétrica e promover o seu uso eficiente, induzindo ações de eficiência energética em diferentes segmentos da economia, que auxiliam o país a economizar energia elétrica. Em 1993 foi instituído o selo Procel (Figura 2) que permite ao consumidor identificar os equipamentos e eletrodomésticos mais eficientes e que consomem menos energia.

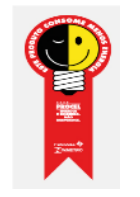

Figura 2 - Selo Procel ( Fonte: www.procelinfo.com.br)

Segundo o Procel (2015), a economia total obtida, no período de 1986 a 2013, foi de 70,1 bilhões de kWh. Os ganhos energéticos anuais mais recentes são exibidos na Figura 3.

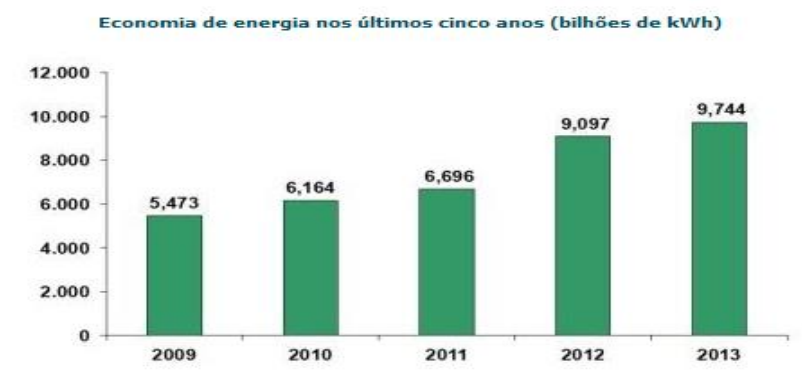

Figura 3: Economia de Energia obtida com o uso do Procel (Fonte: www.procelinfo.com.br).

\subsection{Bandeiras tarifárias}

A partir de janeiro 2015 entrou em vigor em todo o país, exceto no Amazonas, Amapá e Roraima, o sistema de bandeiras tarifárias que indicam o custo para a geração de energia por mês. É um meio de o consumidor entender o custo para a geração de energia fornecida e praticar o consumo consciente.

O sistema conta com três bandeiras: verde, amarela e vermelha. A bandeira verde representa condições favoráveis de geração de energia onde a tarifa não sofre nenhum acréscimo. A bandeira amarela indica condições de geração menos favoráveis onde à tarifa sofre acréscimo de $R \$ 0,025$ para cada quilowattshora (kWh) consumidos. Já a bandeira vermelha indica custos elevados para a geração de energia e a tarifa sofre acréscimo de $R \$ 0,055$ para cada quilowatthora kWh consumidos.

Para usuários de baixa renda existe a possibilidade de obter a Tarifa Social que oferece descontos de 10\% a $65 \%$ sobre a fatura de energia elétrica.

Segundo a Aneel para contar com o beneficio a família precisa está inscrita no Cadastro Único para Programas Sociais do Governo Federal e apresentar renda familiar mensal per capita menor ou igual a meio salário mínimo; ou aqueles cadastrados no Benefício de Prestação Continuada da Assistência Social - BPC, nos termos dos arts. 20 e 21 da Lei no 8.742/1993; ou ainda aqueles cuja família esteja inscrita no Cadastro Único com renda mensal de até 3 (três) salários mínimos, que apresente portador de doença ou deficiência cujo tratamento, procedimento médico ou terapêutico requeira o uso continuado de aparelhos, 
equipamentos ou instrumentos cujo funcionamento demandem consumo de energia elétrica.

\section{A Atividade Matemática}

Para modelagem de atividade matemática proposta devemos entender primeiramente alguns conceitos fundamentais de função, energia e compreensão das variáveis envolvidas no consumo de energia elétrica.

Uma função é uma relação entre duas variáveis $x$ e y tal que o conjunto de valores para $x$ é determinado, e a cada valor $x$ está associado um e somente um valor para y. Essa relação entre $x$ e y é expressa por $y=f(x)$, onde x é o domínio da função e y a imagem da função. Para compreensão de conceitos de energia é necessário que se faça algumas considerações importantes sobre potencia elétrica, corrente e tensão elétrica.

A potencia elétrica pode ser entendida como o trabalho realizado pela corrente elétrica em um circuito simples, é calculado como sendo produto da tensão pela corrente. A unidade para potencia elétrica no sistema internacional de medidas é o Watts com o símbolo W.

A corrente elétrica é o fluxo de elétrons que percorre um circuito quando este é conectando a uma fonte ou uma tensão elétrica. A unidade de corrente elétrica é dada por i segundo o sistema internacional de medidas, enquanto a tensão elétrica é medida em volts e é a diferença de potencial entre dois pontos distintos.

O cálculo de consumo de energia é construído a partir do estudo das seguintes variáveis.

- $\quad$ Potência do equipamento (Watts) $=\mathrm{W}$

- Quantidade de aparelhos (unidade) $=$ Qte

- Tempo de uso diário (horas) = horas

- $\quad$ Dias de uso mês (dias) = dias

- Consumo $(\mathrm{kWh} / \mathrm{mês})=$ Consumo

- $\quad$ Valor da tarifa $(\mathrm{R} \$)=$ Tarifa

Cada equipamento possui especificado pelo seu fabricante ou em seu manual sua potência média, e de acordo com a quantidade de aparelhos pode-se mensurar o gasto pelo tempo de uso, por exemplo, um chuveiro elétrico possui cerca de 5500 watts.

Para o cálculo da tarifa é necessário saber qual o consumo total do mês e verificar no site da ANEEL a tarifa correspondente de acordo com a classificação baixa renda ou tarifa normal. As tarifas podem ser verificadas conforme a figura 4 :

\begin{tabular}{|l|r|}
\hline \multicolumn{1}{|c|}{ Descrição } & R\$/kWh* \\
\hline B1 - Residencial & 0,45312 \\
\hline B1 - Residencial Baixa Renda & \\
\hline Consumo mensal inferior ou igual a $30 \mathrm{kWh}$ & 0,15652 \\
\hline Consumo mensal superior a $30 \mathrm{kWh}$ e inferior ou igual a $100 \mathrm{kWh}$ & 0,26832 \\
\hline Consumo mensal superior a $100 \mathrm{kWh}$ e inferior ou igual a $220 \mathrm{kWh}$ & 0,40248 \\
\hline Consumo mensal superior a $220 \mathrm{kWh}$ & 0,44720 \\
\hline
\end{tabular}

* Os valores constantes da Resolução Homologatória referida são expressos em R\$/MWh

Figura 4: Tarifas da Classe de Consumo Residencial de uma Concessionária (ANEEL, 2015).
A base de cálculo do consumo considera o número de kWh por mês. Um kWh é a quantidade de energia necessária para alimentar um equipamento que possui potência de $1000 \mathrm{~W}$ (watts) num período de uma hora.

Sendo assim, podemos deduzir as seguintes expressões:

Consumo de energia mensal em kWh:

\section{- $\quad$ Consumo $=(\mathrm{W} \times$ Qte $\times$ horas $\times$ dias $) / 1000$}

De acordo com o consumo mensal e sua classificação de tarifa social ou normal, o valor a pagar na conta de energia elétrica $R \$$ é deduzido pela função afim do tipo $f(x)=a x$ :

\section{* Valor=Tarifa $\times$ Consumo}

Por exemplo, vamos calcular o valor da energia a ser pago por utilização de um chuveiro elétrico com potência de 3500 watts, e que é utilizado diariamente por 15 minutos. O calculo ficaria da seguinte maneira:

- $W=3500$ watts;

- Qte= 1 aparelho

- Horas =1 / 4 horas (15 minutos)

- $\quad$ Dias $=30$ dias

- $\quad$ Tarifa $=$ considerando $0,36778 \mathrm{R} \$ / \mathrm{kWh}$

$\checkmark$ Consumo $=(3500 \times 1 \times 1 / 4 \times 30) / 100$

$\checkmark$ Consumo $=26,25 \mathrm{kWh}$

$\checkmark \quad$ Valor $=0,36778 \times 26,25=R \$ 9,65$

O valor total da conta de energia elétrica é deduzido a partir da relação entre as variáveis demonstradas, de acordo com a variação da tarifa e o consumo mensal o valor a ser pago é gerado, ou seja, o valor da prestação do serviço de energia elétrica está diretamente proporcional ao tempo de utilização dos aparelhos elétricos disponíveis nas residências, quem consome mais kWhs paga mais em sua conta de energia elétrica.

\section{Considerações}

Neste artigo, abordamos a modelagem matemática como estratégia pedagógica para o ensino de matemática (função afim) a partir do contexto do consumo de energia elétrica, apontando a necessidade de uma abordagem mais contextualizada e próxima ao cotidiano do aluno. A atribuição de sentido nos conteúdos de matemática contribui de forma significativa para a motivação do aluno e do ambiente escolar. Modelar uma situação problema em sala de aula favorece e dinamiza a produção de conhecimento. 


\section{Referências}

[1] BARBOSA, J. C. Modelagem na Educação Matemática: contribuições para o debate teórico. In: REUNIÃO ANUAL DA ANPED, 24. 2001, Caxambu. Anais... Caxambu: ANPED, 2001. 1 CD-ROM.

[2] SKOVSMOSE, Ole. Cenários de Investigação. In: Revista Bolema, Ano 13, no 14, pp.66 a 91, 2000 ._. Educação Matemática Crítica A questão da democracia Campinas: Papirus, 2011.

[3] BURAK, D. ; ARAGÃO, R.M.R. de . A modelagem matemática e as relações com a aprendizagem significativa. Curitiba, PR: CRV, 2012.

[4] BARBOSA, Maria Hermínio. e BORBA, Marcelo de Carvalho. A Modelagem Matemática e questões sociais em Educação Matemática. -In: Encontro Paulista de Educação Matemática, 8p, 2006, São Paulo. Anais do VIII EPEM - São Paulo. Universidade Cruzeiro do Sul, 2006. CD ROM.

[5] FERRI, Rita B. Theoretical and empirical differentiations of phases in the modeling process. Zentralblatt für Didaktik der Mathematik - ZDM - The International Journal on Mathematics Education, v. 38, n. 2, p. 86-95, 2006.

[6] BASSANEZI, R. C.. Ensino - aprendizagem com modelagem matemática. São Paulo: Editora Contexto, 2002.

[7] PROCEL - Programa Nacional de Conservação de Energia Elétrica. Disponível em $<\mathrm{http}$ ://www.procelinfo.com.br/main.asp?Team $=\{505 \mathrm{FF}$ 883-A273-4C47-A14E-0055586F97FC\}>. Acesso em: 24 abr. 2015.

[8] ANEEL - Energia consciente. Disponível em <http://www.aneel.gov.br/>. Acesso em: 25 abr. 2015. 